\title{
ORIGINAL
}

\section{CUMPLIMIENTO DE LOS OBJETIVOS DE CONTROL METABÓLICO EN DIABETES MELLITUS EN EL MEDIO RURAL DE OURENSE}

Gabriel J Díaz Grávalos (1), Gerardo Palmeiro Fernández (2), Inmaculada Casado Górriz (3), Margarita Arandia García (4), M Maite Portuburu Izaguirre (1) y Luis A Vázquez Fernández (1)

(1) Centro de Salud Cea. Ourense.

(2) Centro de Salud O Barco. Ourense.

(3) Centro de Salud Allariz. Ourense.

(4) Centro de Salud Coles. Ourense.

\section{RESUMEN}

Fundamento: Valorar el cumplimiento de los objetivos de control metabólico en el paciente diabético tipo 2 en la atención primaria de ámbito rural, comparándolo con los valores recomendados por la American Diabetes Association (ADA) y el Grupo de estudio de la diabetes en atención primaria de salud (GEDAPS).

Métodos: Estudio descriptivo transversal. Se calculó un tamaño muestral, basándose en la determinación de la hemoglobina glicosilada (HbA1c), de 119 individuos. Participaron 253 personas diabéticas tipo 2 con al menos 2 años de seguimiento en su centro de salud. Fueron seleccionados de forma aleatoria de 17 cupos médicos de 11 centros de salud rurales de la provincia de Ourense. A partir de la historia clínica se recogieron variables demográficas, factores de riesgo cardiovascular, tratamiento farmacológico, realización de autoanálisis, fondo de ojo, sensibilidad táctil, HbAlc, perfil lipídico, presión arterial e índice de masa corporal.

Resultados: En el 44,3\% de las personas de la muestra se había determinado $\mathrm{HbA} 1 \mathrm{c}$ en los últimos 6 meses y presentaba un valor inferior a $7 \%$. Un $21,2 \%$ tenía la presión arterial por debajo de 130/80, y el 19,8\% un colesterol LDL menor de $100 \mathrm{mg} / \mathrm{dl}$. Entre los pacientes con colesterol LDL mayor de 100 el 40,7\% no recibía tratamiento hipolipemiante. El 20,4\% de los que tenían cifras elevadas de presión arterial no recibía tratamiento hipotensor. Considerando estos tres factores el 2,5\% alcanzaba los 3 objetivos de control. Un $36 \%$ realizaba tratamiento antiagregante con ácido acetil salicilico.

Conclusiones: Se constata un importante déficit tanto en la frecuencia de los controles realizados por los profesionales sanitarios como en el número de intervenciones realizadas para conseguir los objetivos propuestos, con resultados muy inferiores a lo recomendado en las guías de práctica clínica.

Palabras clave: Diabetes mellitus. Atención primaria de salud. Salud rural. Control de calidad.

Correspondencia:

Gabriel J Díaz Grávalos

Feria 9

CEA

32130 Ourense

Correo electrónico: gdgravalos@hotmail.com

\section{ABSTRACT \\ The Diabetes Mellitus Metabolic Check-up Objectives Met in the Rural Area of Ourense, Spain}

Background: To evaluate the degree to which the metabolic check-up objectives among Type II diabetes patients were met in rural primary care by comparing them to the values recommended by the American Diabetes Association (ADA) and the Diabetes in Primary Care Study Group (GEDAPS).

Methods: Descriptive cross-sectional study. A sample size was calculated based on the glycosolated hemoglobin (HbA1c) test in 119 individuals. A total of 253 patients with Type II diabetes followed up for at least two years at their healthcare facility took part. The patients in question were selected at random from among 17 medical lists at 11 rural healthcare facilities in the province of Ourense (Spain). Demographic variables, cardiovascular risk factors, pharmacological treatment, self-analyses, eye fundus, tactile sensitivity, $\mathrm{HbA} 1 \mathrm{c}$, lipid profile, blood pressure and body mass index data were taken from the patients' clinical records.

Results: A total of $44.3 \%$ of those in the sample had undergone an $\mathrm{HbAlc}$ test within the immediately prior six-month period, showing a value of under seven percent $(7 \%)$. A total of $21.2 \%$ had a blood pressure of under 130/80, and $19.8 \%$ a LDL cholesterol level of under $100 \mathrm{mg} / \mathrm{dl}$. A total of $40.7 \%$ of those patients with a LDL cholesterol level over 100 were not undergoing any hypolipemiant treatment. A total of $20.4 \%$ of the patients showing high blood pressure readings were not undergoing any blood pressure lowering treatment. On the basis of these three factors, a total of $2.5 \%$ met the check-up objectives. Thirty-six percent $(36 \%)$ were antiaggregated with AAS.

Conclusions: A major deficit is revealed both in the frequency of the check-ups conducted by the healthcare professionals as well as the number of interventions performed for achieving the proposed objectives, showing results far below what is recommended in the clinical practice guidelines.

Key words: Diabetes mellitus. Primary Health Care. Rural Health. Quality control. 


\section{INTRODUCCIÓN}

La diabetes mellitus tipo 2 (DM2) constituye una de las patologías más frecuentes en la consulta del médico de familia. En los últimos años el número de diabéticos se ha incrementado de forma dramática, achacándose a un conjunto de factores que inciden sobre la población, destacando la ingesta calórica excesiva y la escasa realización de ejercicio físico ${ }^{1}$.

Desde diversos organismos nacionales e internacionales se han diseñado directrices para su diagnóstico temprano y la intervención terapéutica que permita reducir o retrasar la aparición de complicaciones ${ }^{2,3}$. Así, entre otros, tanto la American Diabetes Association (ADA) como el Grupo de estudio de la diabetes en atención primaria de salud (GEDAPS) consideran la consecución de determinados valores en diversos parámetros biológicos como objetivos a conseguir en el tratamiento de la enfermedad. Entre ellos destaca por su importancia el valor de la hemoglobina glicosilada ${ }^{3}$, ya que proporciona información sobre el grado de control de la glucemia en los 3-4 meses previos y se encuentra relacionada con la aparición de complicaciones a largo plazo, además de otros factores de riesgo cardiovascular, como la presión arterial, los lípidos sanguíneos o el índice de masa corporal.

Sin embargo, a pesar de la importante sistematización de las actividades a desarrollar tanto en el tratamiento como en la prevención, el grado de control de la enfermedad dista mucho de ser el óptimo ${ }^{4,5}$.

Como en otras parcelas de la actividad sanitaria, la práctica rural se encuentra en teoría con más dificultades para llevar a cabo estas medidas, debido básicamente a un acceso más dificultoso a los medios técnicos y organizativos necesarios para cumplimentarlas, existiendo estudios en los que los pacientes diabéticos del medio rural presentan peores resultados en sus parámetros de control que en el medio urbano ${ }^{6,7}$. Sin embargo, la diabetes es considerada por los profesionales que ejercen en el medio rural como una de sus prioridades en la práctica diaria $^{8}$.

Con el fin de valorar la calidad de la asistencia prestada al paciente diabético tipo 2 en la atención primaria rural y las posibles áreas de mejora se llevó a cabo una evaluación del cumplimiento de los objetivos de control metabólico anteriormente citados, considerando su realización y el grado de control alcanzado.

\section{SUJETOS Y MÉTODOS}

Se realizó un estudio descriptivo transversal. Con arreglo a los objetivos de control recomendados por la ADA y el GEDAPS ${ }^{2,3}$, se procedió a recoger los datos reflejados en la tabla 1, a partir de las historias clínicas (HC) de los pacientes seleccionados, por medio de sus respectivos médicos de cabecera y de forma anónima.

Se consideró la hemoglobina glicosilada (HbA1c) como el parámetro principal y, a partir del mismo y según los resultados de estudios previos en nuestro país ${ }^{4}$, se determinó un tamaño muestral necesario de 119 individuos para realizar su estimación ( $\left.D E=1,39 ; d= \pm 0,25 ; z_{\alpha}=1,96\right)$. Los valores objetivo de los diferentes parámetros se especifican en tabla 1 .

Se seleccionó a 253 pacientes de 17 cupos médicos en 11 municipios del medio rural ourensano, que suponen unas 18.500 tarjetas sanitarias individuales. Los facultativos que participaron en la selección lo hicieron basándose en su pertenencia a la red de médicos rurales investigadores ourensanos del Grupo de Medicina Rural de semFYC. Se acordó con todos ellos la sistemática de la recogida de datos. Cada facultativo seleccionó a los 15 primeros pacientes diabéticos tipo 2 con más de dos años de antigüedad 
Tabla 1

Datos obtenidos de los pacientes incluidos en el estudio y valores objetivo considerados

\begin{tabular}{|c|c|c|}
\hline Características y Tratamiento & Parámetros de control & Valores objetivo \\
\hline $\begin{array}{l}\text { Edad y Sexo } \\
\text { Factores de riesgo cardiovascular } \\
\text { registrados: } \\
\text { - HTA } \\
\text { - Dislipidemia } \\
\text { - Tabaquismo } \\
\text { - Obesidad } \\
\text { Tratamiento prescrito: } \\
\text { - AAS } \\
\text { - Hipolipemiantes } \\
\text { - IECAs ó ARA II } \\
\text { - Otros hipotensores } \\
\text { - Insulina } \\
\text { - Metformina } \\
\text { - Sulfonilureas }\end{array}$ & $\begin{array}{l}\text { Realización de Autoanálisis } \\
\text { Realización en el último año de: } \\
\text { - Fondo de ojo } \\
\text { - Inspección de los pies (monofilamento) } \\
\text { Realización en los últimos } 6 \text { meses y valor obtenido: } \\
\text { - HbA1c } \\
\text { - Colesterol LDL } \\
\text { - Colesterol HDL } \\
\text { Realización en los últimos } 3 \text { meses y valor obtenido: } \\
\text { - Determinación de presión arterial } \\
\text { - Determinación de IMC }\end{array}$ & $\begin{array}{c}<7 \% \\
<100 \mathrm{mg} / \mathrm{dl} \\
>40 \mathrm{mg} / \mathrm{dl} \\
\\
<130 / 80 \mathrm{mmHg} \\
<27 \mathrm{~kg} / \mathrm{m}^{2}\end{array}$ \\
\hline
\end{tabular}

desde el diagnóstico, según fueron vistos en consulta a lo largo del periodo de recogida de datos (marzo 2004). Con el fin de comprobar la adecuación de la muestra, a partir de los listados del Programa de Diabetes de cinco de los cupos, se obtuvieron los datos de edad y sexo, sin que se encontraran diferencias significativas con la muestra estudiada.

Una vez tabulados los datos, el análisis se realizó con ayuda de los programas informáticos SPSS 12.0 (SPSS Inc) y EPIDAT 3.0 (Xunta de Galicia/OPS/OMS). Los resultados se expresan como media (desviación estándar) y/o porcentaje. Se calcularon los intervalos de confianza del $95 \%\left(\mathrm{IC}_{95 \%}\right)$. Se comprobó la normalidad de los datos antes de realizar pruebas estadísticas que requirieran tal condición. En caso de no cumplirla se utilizaron pruebas no paramétricas. Las pruebas utilizadas (comparación de medias y porcentajes, $\chi^{2}$, correlación) se consideraron significativas con valores de $\mathrm{p} \leq 0,05$ (no significativas $=n s$.). Con el fin de determinar posibles predictores de control entre las variables seleccionadas se realizó un análisis de regresión logística, considerando como variable dependiente el valor de la $\mathrm{HbA}_{1 \mathrm{c}}$, que fue clasificada como buen control/mal control $\left(\mathrm{HbA}_{1 \mathrm{c}}<7 / \geq 7\right)$, y como variables predictoras: edad, sexo, IMC, realización de autoanálisis, número de fármacos antidiabéticos utilizados (insulina, metformina, sulfonilureas) y número de controles realizados por parte de los facultativos. El resultado se expresó en forma de odds ratio (OR) con sus correspondientes intervalos de confianza del $95 \%$.

\section{RESULTADOS}

De los 253 casos que se analizaron, 126 eran varones $(49,8 \%)$ y 127 mujeres $(50,2 \%)$. La edad media era de $69,1(11,2)$ años ( $\sigma^{\star} 68,2$ y 969,9 ; n.s.) con rango entre 39 y 100 . La presencia de factores de riesgo (FR) según figuraba registrada en la historia clínica queda reflejada en la tabla 2 . El $40,3 \%\left(\mathrm{IC}_{95 \%}=34,1-46,5\right)$ de la muestra presentaba más de un FR, sin relación con edad o sexo. 
Tabla 2

Factores de riesgo registrados en las historias clínicas de los pacientes analizados (\% sobre el total)

\begin{tabular}{|l|c|c|c|c|}
\cline { 2 - 5 } \multicolumn{1}{c|}{} & HTA & Dislipidemia & Tabaquismo & Obesidad \\
\hline Varones & $78(61,9 \%)^{*}$ & $58(46.0 \%) \dagger$ & $10(7,9 \%)$ & $27(21,4 \%) \ddagger$ \\
\hline Mujeres & $89(70,1 \%)^{*}$ & $70(55,1 \%) \dagger$ & - & $34(26,8 \%) \ddagger$ \\
\hline Total & $167(66.0 \%)$ & $128(50,6 \%)$ & $10(4,0 \%)$ & $61(24,1 \%)$ \\
\hline
\end{tabular}

* Diferencia no significativa (n.s.), $\dagger$ n.s., $\ddagger$ n.s.

Tabla 3

Número (\%) de pacientes con los controles clínicos realizados y valores promedios observados

\begin{tabular}{|c|c|c|c|}
\hline & Varones & Mujeres & Total \\
\hline Autoanálisis & $64(50,8 \%)$ & $60(47,2 \%)$ & $124(49,0 \%)$ \\
\hline Fondo de ojo & $80(63,5 \%)$ & $79(62,2 \%)$ & $159(62,8 \%)$ \\
\hline Estado pies & $80(63,5 \%)$ & $89(70,1 \%)$ & $169(66,8 \%)$ \\
\hline Presión arterial (realizado) & $114(90,5 \%)$ & $119(93,7 \%)$ & $233(92,1 \%)$ \\
\hline PAS (valor obtenido) & $138,4 \pm 16,7$ & $135,3 \pm 17,6$ & $136,8 \pm 17,2$ \\
\hline PAD (valor obtenido) & $74.0 \pm 11,4$ & $74,4 \pm 9,4$ & $74,2 \pm 10,4$ \\
\hline IMC (realizado) & $80(63,5 \%)$ & $88(69,3 \%)$ & $168(66,4 \%)$ \\
\hline IMC (valor obtenido) & $28,9 \pm 3,5$ & $29,8 \pm 4,8$ & $29,4 \pm 4,2$ \\
\hline $\mathrm{HbA}_{1 \mathrm{c}}$ (realizado) & $98(77,8 \%)$ & $67(84,3 \%)$ & $205(81,0 \%)$ \\
\hline $\mathrm{HbA}_{1 \mathrm{c}}$ (valor obtenido) & $7,08 \pm 1,6$ & $7,22 \pm 1,6$ & $7,15 \pm 1,6$ \\
\hline HDL (realizado) & $77(61,1 \%)$ & $88(69,3 \%)$ & $165(65,2 \%)$ \\
\hline HDL (valor obtenido) & $49,5 \pm 17,7$ & $55,0 \pm 18,3$ & $52,4 \pm 18,2$ \\
\hline LDL (realizado) & $77(61,1 \%)$ & $89(70,1 \%)$ & $166(65,6 \%)$ \\
\hline LDL (valor obtenido) & $121,0 \pm 34,6$ & $119,1 \pm 35,0$ & $120,0 \pm 34,7$ \\
\hline
\end{tabular}


La $\mathrm{HbA}_{1 \mathrm{c}}$ tenía un valor medio de 7,1 $(1,6)\left(\mathrm{IC}_{95 \%},=6,9-7,3\right)$, sin diferencias significativas entre varones y mujeres ni en función de la edad. Entre los 205 pacientes ( $81 \%$ de la muestra) en los que se había determinado la $\mathrm{HbA}_{1 \mathrm{c}}, 54,6 \%\left(\mathrm{IC}_{95 \%}=47,5-\right.$ $61,7)$ tenían valores inferiores a 7\%. Si se considera la totalidad de la muestra, el $44,3 \%$ de los casos $\left(\mathrm{IC}_{95 \%},=37,9-50,6\right)$ tenía una determinación de hemoglobina glicosilada en los últimos 6 meses y dentro de los valores aceptados como buen control.

La tabla 3 resume el número y porcentaje de pacientes con los parámetros de control realizados y los valores promedios observa- dos. No se encontraron diferencias significativas en función del sexo.

La utilización de fármacos queda reflejada en la tabla 4.

Entre los pacientes con colesterol LDL $\geq$ 100 un $40,7 \%\left(\mathrm{IC}_{95 \%},=31,2-50,2\right)$ no tenía prescrito un hipolipemiante. Igualmente, entre los pacientes con cifras de presión arterial por encima del valor objetivo un $20,4 \%$ $\left(\mathrm{IC}_{95 \%},=14,3-26,5\right)$ no tomaba fármacos hipotensores.

Del total de personas incluidas a un $15,4 \%$ $\left(\mathrm{IC}_{95 \%},=11,3-20,5\right)$ se le había realizado la

Tabla 4

Fármacos prescritos entre los diabéticos estudiados

\begin{tabular}{|l|c|c|c|c|}
\cline { 2 - 5 } \multicolumn{1}{c|}{} & Varones & Mujeres & p & Total (\%, IC $95 \%)$ \\
\hline AAS & $47(37,3 \%)$ & $44(34,6 \%)$ & n.s. & $91(36 \% ; 29,8-42,1)$ \\
\hline Hipolipemiantes & $58(46,0 \%)$ & $73(57,5 \%)$ & n.s. & $131(51,8 \% ; 45,4-58,1)$ \\
\hline IECAS/ARA II & $77(61,1 \%)$ & $81(63,8 \%)$ & n.s. & $158(62,5 \% ; 56,3-68,6)$ \\
\hline Otros hipotensores & $31(24,6 \%)$ & $41(32,3 \%)$ & n.s. & $72(28,5 \% ; 22,7-34,2)$ \\
\hline Insulina & $25(19,8 \%)$ & $33(26,0 \%)$ & n.s. & $58(22,9 \% ; 17,5-28,3)$ \\
\hline Metformina & $36(28,6 \%)$ & $31(24,4 \%)$ & n.s. & $67(26,5 \% ; 20,8-32,1)$ \\
\hline Sulfonilureas & $56(44,4 \%)$ & $37(29,1 \%)$ & 0,01 & $93(36,8 \% ; 30,6-42,9)$ \\
\hline
\end{tabular}

Tabla 5

Parámetros de control y porcentaje $\left(\mathrm{IC}_{95 \%}\right)$ de pacientes con objetivo alcanzado

\begin{tabular}{|l|l|}
\hline $\mathbf{H b A}_{\mathbf{1 c}}<\mathbf{7} \%$ & $45,1 \%\left(\mathrm{IC}_{95 \%}, 38,7-51,4\right)$ \\
\hline $\mathbf{P A}<\mathbf{1 3 0} / \mathbf{8 0} \mathbf{~ m m H g}$ & $21,2 \%\left(\mathrm{IC}_{95 \%}, 15,8-26,6\right)$ \\
\hline $\mathbf{L D L}<\mathbf{1 0 0} \mathbf{~} \mathbf{~ g / d l}$ & $19,8 \%\left(\mathrm{IC}_{95 \%}, 14,6-24,9\right)$ \\
\hline $\mathbf{H D L}>\mathbf{4 0} \mathbf{~} \mathbf{~ g} / \mathbf{d l}$ & $51,8 \%\left(\mathrm{IC}_{95 \%, 45,4-58,1)}\right.$ \\
\hline $\mathbf{I M C}<\mathbf{2 7} \mathbf{~ k g} / \mathbf{m}^{\mathbf{2}}$ & $19,8 \%\left(\mathrm{IC}_{95 \%}, 14,6-24,9\right)$ \\
\hline
\end{tabular}


Variables predictoras respecto al control adecuado de $\mathrm{HbA}_{1 \mathrm{c}}$

\begin{tabular}{|c|c|c|c|}
\hline Variable & OR & IC $_{\mathbf{9 5} \%}$ & $\mathbf{p}$ \\
\hline IMC & 0,87 & $0,8-0,9$ & $<0,01$ \\
\hline Número de antidiabéticos prescritos & 0,37 & $0,2-0,6$ & $<0,05$ \\
\hline Número de parámetros de control realizados & 1,32 & $1,1-1,7$ & $<0,01$ \\
\hline
\end{tabular}

totalidad de los ocho controles considerados. Respecto a los resultados el $0,8 \%\left(\mathrm{IC}_{95 \%},=0,1\right.$ 3,0 ) de los pacientes tenía todos los valores numéricos registrados dentro de las cifras recomendadas. En ambos casos no se encontraron diferencias relacionadas con el sexo o la edad. La tabla 5 resume los porcentajes de controles adecuados encontrados. Considerando sólo valores de $\mathrm{HbA}_{1 \mathrm{c}}$, presión arterial y LDL el 2,5\% $\left(\mathrm{IC}_{95 \%},=0,3-4,8\right)$ alcanzaba los tres objetivos de control.

La tabla 6 muestra las variables predictoras con significación estadística en el resultado del análisis de regresión logística tras la transformación en odds del modelo.

\section{DISCUSIÓN}

Respecto a la hemoglobina glicosilada, y según las recomendaciones de $\mathrm{ADA}^{2} \mathrm{y}$ GEDAPS $^{3}$, los resultados obtenidos pueden ser calificados como aceptables en aquellos pacientes en los que había sido determinado, encontrándose en el límite del objetivo de buen control. Sin embargo, casi un $20 \%$ de la muestra no tenía registrada una determinación de este parámetro en los últimos 6 meses, y si a ello se suma el porcentaje de casos con valores iguales o superiores a 7\%, se puede considerar que más de la mitad de los pacientes no tenía un adecuado control de su glucemia o éste no se había realizado dentro del periodo recomendado. La comparación con estudios similares exige conside- rar que no hay uniformidad en los criterios de control respecto a la hemoglobina glicosilada, ni en valores límite ni en frecuencia de controles. Con esta salvedad, estudios similares en el medio rural de varios países muestran unos resultados análogos a los hallados aquí ${ }^{9-11}$. En cuanto al cumplimiento con los intervalos de tiempo establecidos para la determinación de $\mathrm{HbA}_{1 \mathrm{c}}$, el valor obtenido $(81 \%)$ puede considerarse aceptable, y comparable o incluso superior al de encuestas similares $^{9,12}$.

De cualquier forma, múltiples estudios en medio urbano o mixto muestran resultados similares en el control de la glucemia basada en $\mathrm{HbA}_{1 \mathrm{c}}$, y ello tanto entre nosotros ${ }^{5,13}$ como en otros países ${ }^{8,10,12,14-16}$. Al contrario de lo encontrado en otras publicaciones recientes ${ }^{17,18}$ no se han demostrado diferencias relacionadas con el sexo de los pacientes.

La misma reflexión que con la glucemia cabe hacerse respecto a los valores de colesterol LDL, HDL, presión arterial o IMC. Contrastan llamativamente con los porcentajes de factores de riesgo reconocidos a partir de las historias clínicas (tabla 2), lo que hace pensar en un infradiagnóstico de los mismos o en un registro inadecuado, presuponiendo de cualquier forma un déficit de calidad en el proceso de atención.

Numerosos estudios demuestran en el ámbito del tratamiento de la dislipide- 
mia $^{4,14,19-20}$ y de la presión arterial ${ }^{4,9,11,14,20-22}$ tanto la escasez de controles realizados como la pobreza de los resultados obtenidos, sea en medio rural o urbano.

A este respecto constatamos un importante desfase entre la presencia de factores de riesgo y el uso de fármacos para su control, resultados coincidentes en general con los hallados en diversos estudios respecto al escaso uso de hipolipemiantes, hipotensores y ácido acetilsalicílico ${ }^{11,19,21-24}$.

Considerando el conjunto de valores de $\mathrm{HbA}_{1 \mathrm{c}}$, PA y LDL ningún autor encuentra más de un $15 \%$ de pacientes que consigan resultados adecuados $9,11,12,20$, cifra que en este estudio no alcanza el 3\%, fundamentalmente a expensas de un escaso control de la dislipemia. Merecen destacarse las elevadas cifras de IMC obtenidas, sobre todo por la dificultad de manejo de este FR.

En cuanto al uso de monofilamento y exploración de fondo de ojo los resultados son básicamente concordantes también con lo obtenido por otros autores, aunque aquí la variabilidad es más acusada ${ }^{9,25,26}$.

La realización de autoanálisis no se relaciona en nuestro estudio con mejores resultados de $\mathrm{HbA}_{1 \mathrm{c}}$. Obviamente, puede deberse a que los pacientes que lo realizan son aquellos que tienen más dificultad para conseguir valores adecuados, pero de cualquier modo creemos que sería necesario constatar de forma fehaciente la utilidad del autoanálisis rutinario en el paciente tipo 2 en tratamiento con dieta y/o antidiabéticos orales, porque probablemente los resultados no compensan los recursos invertidos.

Los resultados obtenidos en el análisis multivariante entran dentro de lo esperado, con mejores valores de $\mathrm{HbA}_{1 \mathrm{c}}$ asociados a menor IMC y al número de fármacos antidiabéticos utilizados, y peores al número de controles realizados por los facultativos, probablemente porque en este caso se trata de pacientes con diabetes más grave y/o con más dificultad de controlar y, por tanto, sometidos a un seguimiento más intensivo. En cualquier caso, algún autor ha apuntado la posibilidad de que aumentar la frecuencia de los controles no depare mejores resulta$\operatorname{dos}^{27}$.

Entendemos que las principales limitaciones del estudio derivan del método de selección y de la representatividad de los pacientes incluidos.

Si se acepta la premisa de que los médicos participantes están más motivados que la media (pertenencia a un grupo de investigación, interés por la enfermedad diabética), los resultados obtenidos mostrarán, en buena lógica, una situación más favorable a la que pueda darse en el conjunto de los facultativos, lo que deberá ser tenido en cuenta a la hora de generalizar los resultados.

Respecto a la representatividad de los pacientes incluidos en el estudio, estimamos que su selección al azar, junto a su diferente procedencia, hacen que sean lo suficientemente representativos, sin que haya ninguna característica especial que permita diferenciarlos del conjunto de la población diabética rural atendida en sus centros de salud.

Aunque la microalbuminuria se ha constituido como un valor importante en el control del enfermo diabético, no se recogió en nuestro estudio, al igual que la glucemia postprandial. En buena medida, la elección de la $\mathrm{HbA}_{1 \mathrm{c}}$ como único parámetro de control glucémico se realizó basándose en la posibilidad de comparación con estudios similares.

La ausencia de los datos estudiados en las historias clínicas no significa necesariamente que no hayan sido obtenidos e incluso valorados. Esto podría conducir a infravalorar los resultados reales de la atención prestada, pero si aceptamos como indicador de la calidad de la atención la presencia de los 
mismos, es este dato el que debemos considerar. Del mismo modo, tampoco su presencia significa que se haya realizado alguna intervención cuando era necesario hacerlo.

Aceptando la validez del adecuado control de estos parámetros como variables de proceso necesarias para conseguir una disminución de la tasa de enfermedad micro y macrovascular en el diabético, se aprecia un amplio desfase, tanto en frecuencia de intervenciones como en uso de farmacoterapia, que sería conveniente subsanar. Las importantes incertidumbres respecto a cómo conseguirlo $^{28}$ exigen una profundización en la investigación que permita concretar las causas reales de este desfase y las posibles estrategias de solución.

\section{BIBLIOGRAFÍA}

1. American Diabetes Association. Report of the Expert Committee on the Diagnosis and Classification of Diabetes Mellitus. Diabetes Care 2004; 27 (Suppl 1):S5-10.

2. American Diabetes Association. Standards of Medical Care in Diabetes. Diabetes Care 2005; 28 (Suppl 1): S4-36.

3. Franch Nadal J, Goday Arno A, Mata Cases M. COMBO. Actualización 2004. Criterios y pautas de terapia combinada en la diabetes tipo 2. Av Diabetol 2004; 20: 77-112.

4. de la Calle H, Costa A, Díez-Espino J, Franch J, Goday A. Evaluación del cumplimiento de los objetivos de control metabólico de la diabetes mellitus tipo 2. Estudio TranSTAR. Med Clin (Barc) 2003; 120:446-50.

5. Benito López P, García Mayor R, Puig Domingo M, Mesa Manteca J, Pallardo Sánchez LF, Faure Nogueras E et al. Perfil de los pacientes con diabetes mellitus tipo 2, en la Atención Primaria española Rev Clin Esp 2004; 204:1-2.

6. Andrus MR, Kelley KW, Murphey LM, Herndon KC. A comparison of diabetes care in rural and urban medical clinics in Alabama. J Community Health 2004; 29:29-44.

7. Samann A, Kaiser J, Hunger-Dathe W, Schiel R, Muller UA. Population-based measurement of qua- lity of diabetes care using HbA1c values in the state of Thuringia/Germany. Exp Clin Endocrinol Diabetes 2004; 112:531-7.

8. Gamm L, Hutchison L. Rural health priorities in America: where you stand depends on where you sit. J Rural Health. 2003; 19:209-13.

9. Coon P, Zulkowski K. Adherence to American Diabetes Association standards of care by rural health care providers. Diabetes Care 2002; 25:2224-9.

10. Schiel R, Hoffmann A, Muller UA. Behandlungsqualitat bei Patienten mit Diabetes mellitus in einem landlichen Gebiet Deutschlands. Ergebnisse einer Populationsuntersuchung. Die ZEUVIN-Studie. Med Klin (Munich) 1999; 94: 127-32.

11. Toth EL, Majumdar SR, Guirguis LM, Lewanczuk RZ, Lee TK, Jonhson JA. Compliance with clinical practice guidelines for type 2 diabetes in rural patients: treatment gaps and opportunities for improvement. Pharmacotherapy 2003; 23:659-65.

12. Jaboureck O, Mounier-Vehier C, Devos P, Lions C, Dequiedt P, Beregi JP et al. Différences entre recommandations de prise en charge et stratégie globale médicale dans l'hypertension artérielle avec désordres métaboliques en France en 1999. Étude Ohara. Arch Mal Cœur Vaiss 2001 ; 94 : 8348.

13. Virgós M, Castro M, Martínez S, Vila A. ¿Hacemos caso del resultado de la hemoglobina glicosilada a la hora de tratar a nuestros pacientes diabéticos? Aten Primaria 2002; 30:157-62.

14. Saydah SH, Fradkin J, Cowie CC. Poor control of risk factors for vascular disease among adults with previously diagnosed diabetes. JAMA 2004; 291:335-42.

15. Kirk JK, Poirier JE, Mattox MG, Thomas PM, Michielutte R. Compliance with national guidelines in patients with diabetes in a family practice clinic. Pharmacotherapy 2002; 22: 1541-6.

16. Beaton SJ, Nag SS, Gunter MJ, Gleeson JM, Sajjan SS, Alexander CM. Adequacy of glycemic, lipid, and blood pressure management for patients with diabetes in a managed care setting. Diabetes Care 2004; 27:694-8.

17. Hippisley-Cox J, O’Hanlon S, Coupland C. Association of deprivation, ethnicity, and sex with quality indicators for diabetes: population based survey of 53000 patients in primary care. BMJ 2004; 329:1267-70. 
18. Nilsson PM, Theobald H, Journath G, Fritz T. Gender differences in risk factor control and treatment profile in diabetes: a study in 229 swedish primary health care centres. Scand J Prim Health Care 2004; 22:27-31.

19. Mehler PS, Esler A, Estacio RO, McKenzie TD, Hiatt WR, Schrier RW. Lack of improvement in the treatment of hyperlipidemia among patients with type 2 diabetes. Am J Med 2003; 114:37782.

20. McFarlane SI, Jacober SJ, Winer N, Kaur J, Castro JP, Wui MA et al. Control of cardiovascular risk factors in patients with diabetes and hypertension at urban academic medical centers. Diabetes Care 2002; 25:718-23.

21. Supina AL, Guirguis LM, Majumdar SR, Lewanczuk RZ, Lee TK, Toth EL, Johnson JA. Treatment gaps for hypertension management in rural Canadian patients with type 2 diabetes mellitus. Clin Ther 2004; 26: 598-606.

22. Chamontin B, Lang T, Vaisse B, Nicodeme R, Antoine MP, Lazorthes ML et al. Hypertension artérielle et facteurs de risque cardiovasculaires associés chez les diabétiques. À propos de l'enquête PHARE menée en médecine générale. Arch Mal Cœur 2001 ; 94 : 869-73.
23. Esmatjes E, Castell C, Franch J, Puigoriol E, Hernáez R. Consumo de ácido acetilsalicílico en pacientes con diabetes mellitus. Med Clin 2004; 122:96-8.

24. Klinke JA, Johnson JA, Guirguis LM, Toth EL, Lee TK, Lewanczuk RZ, Majumdar SR. Underuse of aspirin in type 2 diabetes mellitus: prevalence and correlates of therapy in rural Canada. Clin Ther 2004; 26: 439-46.

25. Harris SB, Stewart M, Brown JB, Wetmore S, Faulds C, Webster-Bogaert $\mathrm{S}$ et al. Type 2 diabetes in family practice. Room for improvement. Can Fam Physician 2003; 49: 778-5.

26. Chin MH, Auerbach SB, Cook S, Harrison JF, Koppert J, Jin L et al. Quality of diabetes care in community health centers. Am J Public Health 2000; 90:431-4.

27. Grant RW, Buse JB, Meigs JB for the University HealthSystem Consortium (UHC) Diabetes Benchmarking Project Team. Quality of diabetes care in U.S. academic medical centers. Diabetes Care 2005; 28:337-442.

28. Renders CM, Valk GD, Frense LV, Schellevis FG, van Eijk JT, van der Wal G. Long-term effectiveness of a quality improvement program for patients with type 2 diabetes in general practice. Diabetes Care 2001; 24:1365-70. 